\title{
Diffusion modelling on a dynamically adaptive tree grid for rainfall overland flow simulation
}

\author{
Anurak Busaman ${ }^{\mathrm{a}, \mathrm{b}, *}$, Khamron Mekchay ${ }^{\mathrm{a}}$, Suchada Siripant ${ }^{\mathrm{a}}$, Somporn Chuai-Aree ${ }^{\mathrm{b}}$ \\ a Department of Mathematics and Computer Science, Faculty of Science, Chulalongkorn University, \\ Bangkok 10330 Thailand \\ b Department of Mathematics and Computer Science Faculty of Science and Technology, \\ Prince of Songkla University, Muang, Pattani 94000 Thailand
}

*Corresponding author, e-mail: anulove108@hotmail.com

Received 29 May 2015

Accepted 18 Nov 2015

\begin{abstract}
In this study a numerical algorithm is developed for simulation and visualization of rainfall overland flows on natural topography. The algorithm is constructed based on the diffusion model of water flow which is solved numerically using an adaptive tree grid finite volume method which is equipped with dynamic domain defining. The developed algorithm is tested to simulate and visualize rainfall overland flows on natural topography showing the advantages of the algorithm in terms of computational costs and the simulation times without losing much accuracy in the results.
\end{abstract}

KEYWORDS: finite volume method, dynamic domain defining method, visualization

MSC2010: 65C20 68U20 76R50 80M12 76M12

\section{INTRODUCTION}

Rainfall overland flow modelling and simulations are important tools for a flood risk assessment in order to predict areas of flooding that often occur after heavy rainfall. Rainfall overland flow simulation and visualization usually involves numerical methods for solving the model equations for water flows. Most researchers have used shallow water equations to model the rainfall overland flow simulations. For instance, Delestre ${ }^{1}$ developed the rainfall overland flow model using finite volume methods (FVM) for solving 1D shallow water equations. However, several flood models have been successfully developed using simpler equations to reduce complexity of algorithm. For example, Xing $^{2}$ developed a fast method for hydraulic erosion simulation. They used a pipe model to simulate the water movement where the flow velocity is updated by the hydrostatic pressure difference between the two cells connected by the pipe. The model results can simulate the water flow and erosion in rugged terrain. Wang ${ }^{3}$ used a non-zero inertial model to reduce the complexity of the shallow water equations for simulation of flooding in urban areas. The non-zero inertial results agree closely with the shallow water equations. Santillana $^{4}$ analysed and simulated numerically a diffusive wave approximation of the shallow water equations for water overland flow. Dottori ${ }^{5}$ developed and simulated a flood inundation model based on cellular automata.

The motivation of these models are that solving simpler equations should reduce the computational burden and the simulation run times ${ }^{5}$. Simulation and visualization of rainfall overland flows on natural topography requires a large amount of digital terrain data grid cells in order to improve accuracy of the simulation. One general idea is to employ an adaptive tree grid technique, where the methods continuously adjust grid resolution to follow features in the water flow. George ${ }^{6}$ used FVM with block-structured dynamic adaptive mesh refinement in solving $2 \mathrm{D}$ shallow water equations for floods modelling in rugged terrain. Liang $^{7}$ solved 2D shallow water equations with adaptive quad tree grid using FVM for flood inundation model, while Popinet $^{8}$ developed the tsunami model using 2D shallow water equations with a quad tree adaptive grid based on a generalization of the well-balanced positivity-preserving scheme of Audusse ${ }^{9}$. An alternative solution for reducing computational cells is to use the dynamic domain defining method (DDM) presented by Yamaguchi ${ }^{10}$ in the development of GIS-based flood simulation software and applica- 


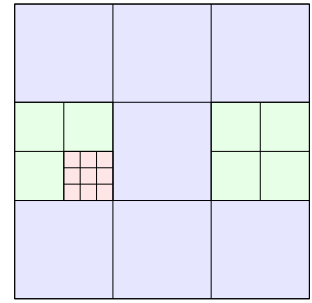

Fig. 1 Regularized tree grid discretization.

tion to flood-risk assessment. The dynamic DDM automatically expands or shrinks the computational domains during the simulation to exclude dry grid cells.

In our previous work ${ }^{11}$, we developed a numerical algorithm in order to simulate and visualize rain-water overland flow. The algorithm is based on a well-balanced, positivity-preserving second order scheme of Audusse ${ }^{9}$ and equipped with the dynamically adaptive general rectangular tree grid method and dynamic DDM for shallow water equations. The results showed that the algorithm is very efficient in terms of computational costs. In this work, by the motivation from Ref. 2, we see that the simulation of rain water overland flow can be performed using equations or algorithms simpler than solving shallow water equations. If the simple equations or algorithms are implemented, the dynamically adaptive general rectangular tree grid algorithm for rain-water overland flow simulation can be developed more easily and has usefulness in terms of computational costs.

The purpose of this work is to develop an algorithm based on adaptive grid finite volume method in solving diffusion equations for water flows in order to simulate and visualize rainfall overland flows on natural topography. The adaptivity is developed based on general rectangular tree grids and in combination with the dynamic DDM.

\section{TREE GRIDS}

The data structure for tree grid was designed for adaptivity based on the work of Popinet ${ }^{8}$ and Liang ${ }^{7}$ that used regularized quad tree grids where each parent cell must have zero or four children and must be balanced in the sense that the levels of adjacent cells cannot differ by more than one.

In order to modify this idea to use with data obtained from natural topography, parent cells can have zero or any number of children cells with square dimension, where parents in the same tree level must have zero or the same number of chil-

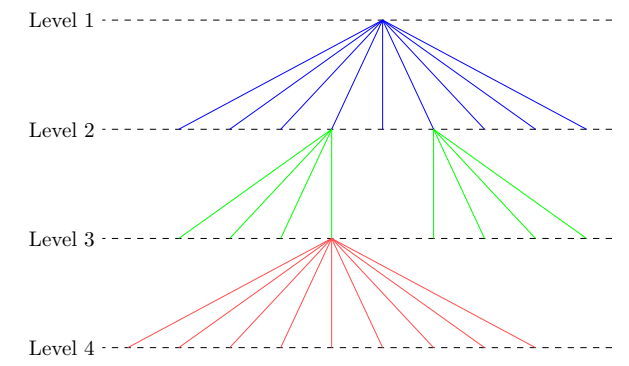

Fig. 2 Hierarchical data structure.

dren, i.e., the parents in level $l$ can have $n^{l} \times n^{l}$ children. In addition, the tree grid does not have to be balanced, i.e., the levels of adjacent cells can differ by more than one. For example, Fig. 1 shows a regularized grid with 4 subdivision levels having 1 big cell in level 1 , and number of children for cells in levels 1,2 , and 3 are $3 \times 3,2 \times 2$, and $3 \times 3$, respectively, while the level 4 cell has no child. During the grid generation process, the corresponding hierarchical tree structure is simultaneously created as illustrated in Fig. 2.

\section{THE MATHEMATICAL MODEL}

In this work, we develop a two-dimensional model for water flow based on diffusion equations for determining the behaviour of rainfall flow above bottom elevation without infiltration. The model is written as

$$
\partial_{t} H=\partial_{x} f+\partial_{y} g+R
$$

where $H=z+h$ is the free surface elevation, $h$ is the water depth, $z$ is the height of topography, $R$ is the rainfall rate, while

$$
f=\alpha \partial_{x} H, g=\alpha \partial_{y} H
$$

are fluxes in the directions of the $x$ and $y$ coordinate axis, respectively. In these equations, $\alpha$ is the water diffusion coefficient and $t$ is time.

\section{NUMERICAL SCHEME}

We present in this section the numerical solution to the model equations described in the previous section. The solution can be generated using conservative finite volume methods.

\section{Finite volume formulation}

The finite volume formulation is performed on the physical domain that is comprised of regularized cells. Each cell is considered as a main control volume whose boundary is formed by the direct 


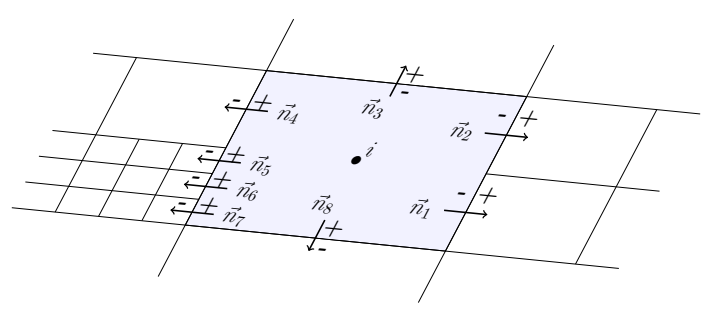

Fig. 3 The main control volume and descriptions for each cell.

walls surrounding it. An example of a single main control volume is shown in Fig. 3.

By integrating (1) over an arbitrary cell domain $\Omega$ and using Green's theorem, we have

$$
\int_{\Omega} \partial_{t} H \mathrm{~d} \Omega=\oint_{\tau} \mathbf{F} \cdot \mathbf{n} \mathrm{d} \tau+\int_{\Omega} R \mathrm{~d} \Omega
$$

where $\tau$ is boundary of $\Omega, \mathbf{n}$ is unit outward normal vector to the boundary, and $\mathbf{F}=(f, g)$ is the vector that consists of the fluxes in (2) at each interface of the cell boundary.

Dividing (3) by the cell area, and calling $H_{i}$ and $R_{i}$ the average of the free surface elevation and rainfall rate interior of the cell, respectively, the finite volume formulation is

$$
\partial_{t} H_{i}=\frac{1}{\Delta x_{i} \Delta y_{i}} \oint_{\tau} \mathbf{F} \cdot \mathbf{n} \mathrm{d} \tau+R_{i}
$$

where $\Delta x_{i}$ and $\Delta y_{i}$ are the widths of the cell wall in the $x$ and $y$ directions, respectively. The subscript $i$ is the spatial index of the cell.

Since our model is performed on dynamically adaptive grid considered as non-uniform grid (by non-uniform grid, each cell can possibly have many adjacent cells and with different size for each wall), the line integral in (4) can be discretized by substituting with the sum over sub-interfaces around the control volume of $\mathbf{F} \cdot \mathbf{n}$ as

$$
\partial_{t} H_{i}=\frac{1}{\Delta x_{i} \Delta y_{i}} \sum_{k} \mathbf{F}_{k} \cdot \mathbf{n}_{k} \Delta \tau_{k}+R_{i}
$$

where the subscript $k$ is the index for each of the sub-interfaces over the boundary of $\Omega$ between the cell and its neighbour, and $\Delta \tau_{k}$ is the width of the sub-interface.

By application of Euler's method to (5), the finite volume formulation over each rectangular cell of the grid is given by

$$
H_{i}^{t+\Delta t}=H_{i}^{t}+\frac{\Delta t}{\Delta x_{i} \Delta y_{i}} \sum_{k} \mathbf{F}_{k} \cdot \mathbf{n}_{k} \Delta \tau_{k}+\Delta t R_{i}^{t}
$$

where $\Delta t$ is time step size.

\section{Numerical flux}

In the developed algorithm, an approximation of fluxes between adjacent cells through each subinterface for simulation of the rainfall flow in rugged terrain is given by

$f_{k}=\alpha \partial_{x} H \approx \alpha \frac{\hat{H}_{+}-\hat{H}_{-}}{\Delta x}, g_{k}=\alpha \partial_{y} H \approx \alpha \frac{\hat{H}_{+}-\hat{H}_{-}}{\Delta y}$

with the Riemann states of water depth

$$
\hat{H}_{ \pm}=\max \left(0, H_{ \pm}^{t}-\max \left(z_{+}, z_{-}\right)\right)
$$

This was also presented by Audusse et $\mathrm{al}^{9}$ in the context of hydrostatic reconstruction. The marker $(-)$ and $(+)$ are indices for the left and right cells at each sub-interface in the $x$ direction while for the $y$ direction, the signs $(-)$ and $(+)$ indicate the bottom and top cells at each sub-interface.

\section{Stability condition}

In order to have a stable scheme as proposed in Ref. 2, the time step size is limited by the condition which is defined by

$$
\Delta t \leqslant 0.25 \frac{\left(\Delta A_{\min }\right)^{2}}{\alpha}
$$

where $\Delta A_{\text {min }}=\min _{\forall i}\left\{\Delta x_{i}, \Delta y_{i}\right\}$ is the minimum dimension of all cells in the computational domain.

\section{Boundary condition}

The opened and closed boundary conditions used in the algorithm are defined as follows. The open boundary condition can be given by

$$
h_{m}=0, \quad z_{m}=0
$$

while the closed boundary condition are given by

$$
h_{m}=0, \quad z_{m}=z_{\text {large }} .
$$

In these equations, the subscript $m$ refers to the spatial index of the computational domain boundary cells, and $z_{\text {large }}$ is some large constant value for the topography height, for example, we assign $z_{\text {large }}=$ 9999.

\section{Grid adaptivity method}

In this developed algorithm, the refinement criterion for the grid adaptivity is checked based on the two conditions as indicators. The first condition is the gradient of the free surface elevation, $H=h+z$. 
A cell should be refined when it has at least one neighbour cell that satisfies

$$
|\nabla H| \Delta_{l}>\epsilon,
$$

where $\nabla H$ and $\Delta_{l}$ are the free surface gradient and the distance between the checked cell and the neighbour cell, and $\epsilon$ is an adjustable parameter (as proposed in Ref. 8). However, since our work is the simulation of the rainfall overland flow, the first condition may be true even when the checked cell has no or very small water depth. Thus $|\nabla(h+z)| \Delta_{l}>$ $\epsilon$ can hold even when $h=0$ or $h$ is very small. This motivates the second condition for the grid adaptivity. The cell should be refined when the checked cell or at least one of its neighbour cells satisfies

$$
h>\xi,
$$

where $\xi$ is an adjustable parameter. With this criterion, a cell is refined whenever both conditions hold. When the cell is refined, the children cells are created in the next level. The water depth of each child is assigned by

$$
h_{j}^{l+1}=h_{i}^{l}
$$

where $h_{j}^{l+1}$ is the water depth of the child of level $l+1$ cell at position $j$, and $h_{i}^{l}$ is the water depth of the parent cell at $i$. The values of height of topography, $z$, are defined by the digital terrain data for each levels as described in the next section.

For the grid adaptivity efficiency, the refined cell should be coarsened when the cells have no need for the calculation. To check for coarsening, all children cells with the same parent are assumed to be coarsened by averaging all the children's values and re-checking the conditions for the refinement. The children cells are destroyed and combined into their parent if the refinement conditions do not hold, and the new water depth of the parent coarsened cell is re-assigned by

$$
h_{i}^{l}=\frac{1}{n_{i}^{l} \times n_{i}^{l}} \sum_{j \in \Omega_{i}} h_{j}^{l+1},
$$

where $n_{i}^{l} \times n_{i}^{l}$ is number of the children of the parent cell.

\section{Topography interpolation}

In real applications of the simulation and visualization of rainfall overland flows, the values of the heights of the topography are obtained by digital terrain data grids. Since the computational grid resolution is possibly different (finer or coarser) than the digital terrain data grid resolution, topography interpolation is required. We present here a method for the topography interpolation using a triangular planes approximation.

For the approximation of the value of the cell in the domain at $i$ :

$$
z_{i}=\left\{\begin{array}{r}
z_{x^{\prime}, y^{\prime}}+\left(z_{x^{\prime}+1, y^{\prime}}-z_{x^{\prime}, y^{\prime}}\right)\left(m-x^{\prime}\right) \\
+\left(z_{x^{\prime}, y^{\prime}+1}-z_{x^{\prime}, y^{\prime}}\right)\left(n-y^{\prime}\right), \\
m \leqslant x^{\prime}-n+y^{\prime}+1, \\
z_{x^{\prime}+1, y^{\prime}+1} \\
+\left(z_{x^{\prime}+1, y^{\prime}}-z_{x^{\prime}+1, y^{\prime}+1}\right)\left(m-\left(x^{\prime}+1\right)\right) \\
+\left(z_{x^{\prime}+1, y^{\prime}}-z_{x^{\prime}+1, y^{\prime}+1}\right)\left(n-\left(y^{\prime}+1\right)\right), \\
m>x^{\prime}-n+y^{\prime}+1,
\end{array}\right.
$$

where $\left(x^{\prime}, y^{\prime}\right)$ is the position of the topographic data grid cell used for approximation with $x^{\prime}=\lfloor m\rfloor$ and $y^{\prime}=\lfloor n\rfloor$,

$$
m=x_{i} n^{x^{\prime}} / n^{x}, \quad n=y_{i} n^{y^{\prime}} / n^{y}
$$

are the mapped indices of $\left(x_{i}, y_{i}\right)$ to the data grid, where $\left(x_{i}, y_{i}\right)$ is the spatial index of the computational grid cell, $n^{x^{\prime}}$ and $n^{y^{\prime}}$ are numbers of columns and rows of the topographic data grid, and $n^{x}$ and $n^{y}$ are numbers of columns and rows of the finest computational grids.

\section{Dynamic domain defining method}

In order to improve the computational efficiency, the dynamic DDM is equipped with the adaptive algorithm. The idea of the dynamic DDM is that each cell $i$ is checked to see if it is necessary for the computation by three conditions as follows. The cell is excluded from the computational domain (the calculation area is shrunk) if the following three conditions are all satisfied: (i) the cell is dry cell, i.e., $h_{i}^{t} \leqslant 0$; (ii) all the neighbour cells are dry, i.e., $h_{k}^{t} \leqslant 0$ for all neighbours $h_{k}^{t}$ of the cell $i$; (iii) the cell has no rainfall, i.e., $R_{i}^{t} \leqslant 0$. The reason of adopting these checks is that when the cell and its neighbour cells are all dry cells, the fluxes of flowing are zero and then the cell water depth is zero. Thus the numerical computation is not necessary for the cell.

\section{Algorithm overview}

We present here an algorithm overview based on the numerical method techniques described above. The developed algorithm consists of many steps of calculations (Fig. 4) and the details are given as follows. 


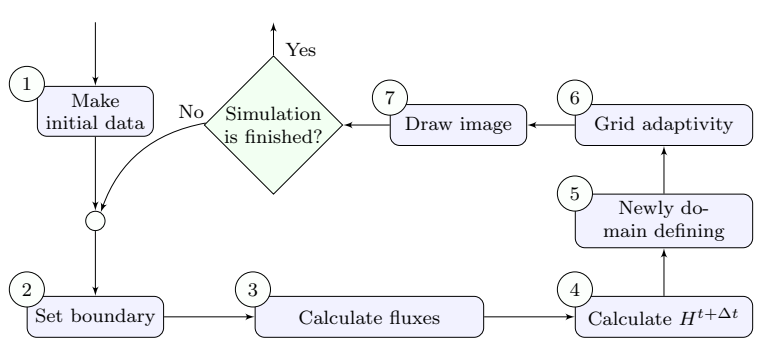

Fig. 4 The algorithm overview.

Step 1: Set the initial values, the cell sizes, number of children in each level, and the maximum level of tree grids.

Step 2: Set the computational grids boundaries. Step 3: Calculate the numerical fluxes and $\Delta t$. Step 4: Calculate $H^{t+\Delta t}((6))$.

Step 5: Define a new domain as the dynamic DDM. Step 6: Conduct the grids adaptivity.

(I) Refinement procedure.

(i) For each cell that is not at the maximum level

(a) Mark cells that satisfy the refinement conditions.

(i) For each marked cells

(a) Create new children cells and assign the dependent values ((13))

(II) Coarsening procedure.

(i) For each parent cell that children have no child

(a) Average the children values as (14);

(b) Check the refinement criterion based on the average values;

(c) If the refinement criterion is false, merge all children cells into a parent cell and assign the dependent values.

Step 7: Visualize the solutions in 2D and 3D by OpenGL.

Step 8: Repeat steps $2-7$ until the simulation is finished.

\section{RESULTS}

The simulations consist of two tests: the rainfall overland flow simulation compared with the simulations using the shallow water equation, and the simulation of rainfall overland flow compared the performance with the simulation on the finest grid. The first numerical simulation is for accuracy and efficiency tests of the developed diffusion model, while the second simulation is for accuracy and efficiency tests of the adaptive tree grid technique.
The coding and simulations are performed using $2 \mathrm{GHz}$ Intel Core i7 with $6 \mathrm{~GB}$ of RAM.

\section{Accuracy and efficiency of the diffusion model}

We illustrate in this section the accuracy and efficiency tests of the diffusion model, which are tested by comparing with the simulation using the shallow water equations. The shallow water equation is simulated using the second order finite volume scheme in Refs. 1,9 with a Manning's coefficient of 0.001. The simulation of the diffusion model is performed on the domain of digital terrain data $7200 \mathrm{~m} \times 7200 \mathrm{~m}$, generated from Shuttle Radar Topography Mission (SRTM) data source with $45 \mathrm{~m}$ $\times 45 \mathrm{~m}$ resolution. Hence the initial grid has $160 \times$ 160 cells. The numerical experiment is simulated for $10000 \mathrm{~s}$ with a water diffusion coefficient of $500 \mathrm{~m}^{2} / \mathrm{s}$. The rainfall rate is set at $0.001 \mathrm{~m} / \mathrm{s}$ within the rectangular regions where $2700 \mathrm{~m} \leqslant x \leqslant$ $4500 \mathrm{~m}$ and $2700 \mathrm{~m} \leqslant y \leqslant 4500 \mathrm{~m}$ for the duration of $3600 \mathrm{~s}$. We used closed boundary conditions.

The 3D representations of the rainfall overland flow simulation for this experiment are presented at different times (Fig. 5). The small pictures in Fig. 5 show the corresponding 2D images. The results show that the diffusion model with the developed numerical scheme has a potential for the simulation of the water flow in rugged terrain.

To check the accuracy and efficiency of the diffusion model with the developed numerical scheme, we found errors of the numerical flooded area of the diffusion model compared with the numerical flooded area using the shallow water equations.

A fit-statistic method, $F$, introduced by Horritt $^{12}$, is used to estimate the ratio between the numerical flooded area and the observed inundated area. Here, $F$ is used to estimate the percentage of the coincided flood extent between the current prediction and the benchmark, which can be described by

$$
F=\frac{W_{\mathrm{c}} \bigcap W_{\mathrm{b}}}{W_{\mathrm{c}} \bigcup W_{\mathrm{b}}}
$$

where $W_{\mathrm{c}}$ and $W_{\mathrm{b}}$ stand for the number of inundated cells given by the present simulation and the benchmark solution, respectively.

$F$ can be specifically used to calculate the error of the model results using

$$
E=\left(1-\frac{\sum_{i} P_{i}^{B_{1} C_{1}}}{\sum_{i} P_{i}^{B_{1} C_{1}}+\sum_{i} P_{i}^{B_{1} C_{0}}+\sum_{i} P_{i}^{B_{0} C_{1}}}\right)
$$

where $P_{i}^{B_{1} C_{1}}$ is 1 when the benchmark cell is wet and the present compared cell is wet. Otherwise 


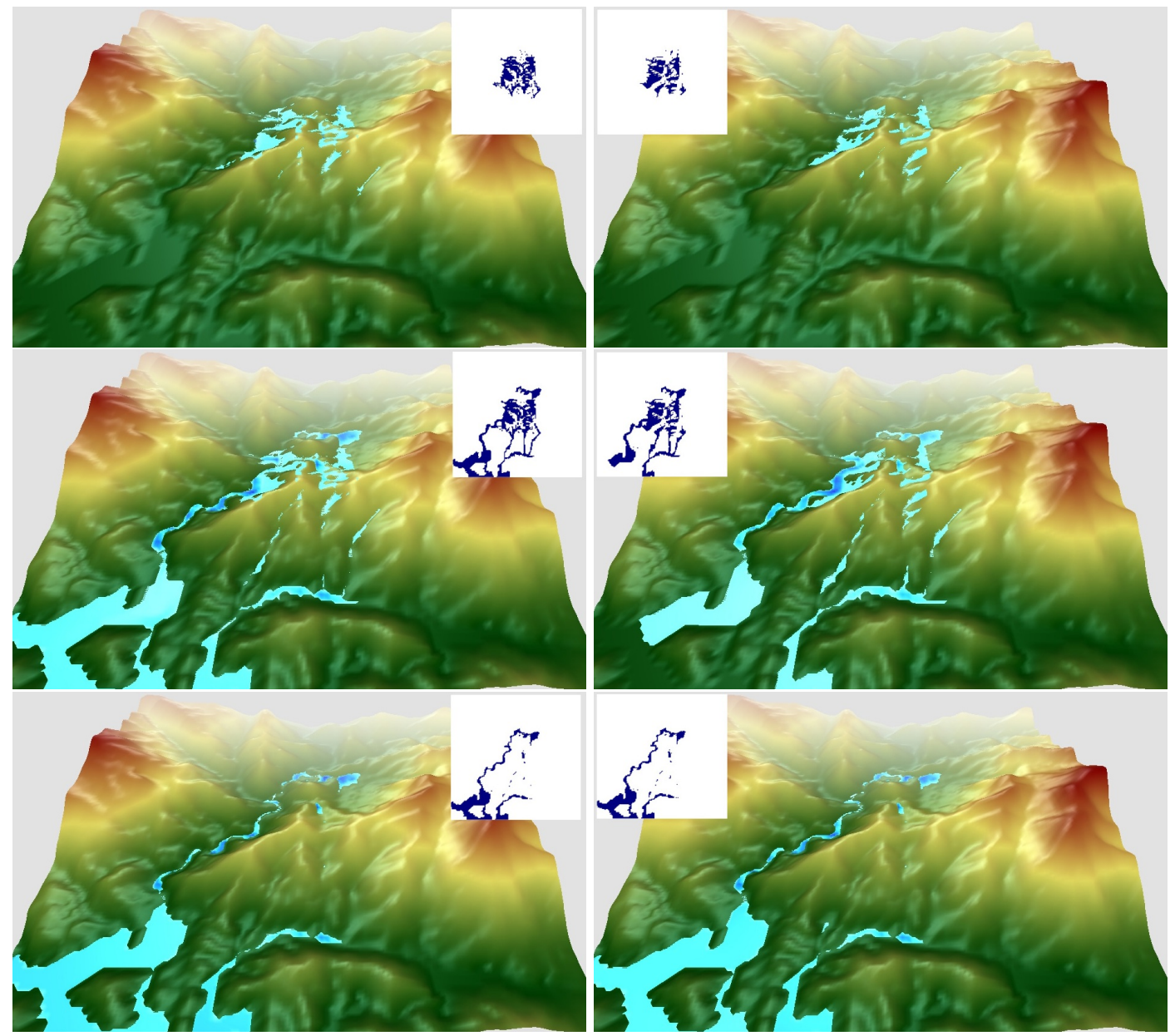

Fig. 5 The 3D representation of the rainfall overland flow simulation using the shallow water equations (left) and the diffusion model (right) at $t=80,3600$, and $10000 \mathrm{~s}$ (from top to bottom).

its value is $0 . \quad P_{i}^{B_{1} C_{0}}$ is 1 when the benchmark cell is wet and the present compared cell is dry; otherwise it is $0 . P_{i}^{B_{0} C_{1}}$ is 1 when the benchmark cell is dry and the compared cell is wet; otherwise its value is 0 . In (18), the numerator accounts for the coincided inundated area predicted by both sets of solutions. The denominator gives the sum of the flooding area defined by either the compared simulation or the benchmark. Hence $E$ is a variable between 0 and 1 . 1 represents the wet-dry state in the compared solution is totally different from the benchmark lattices while 0 represents that the predicted flooding extent is absolutely the same as that in the benchmark lattices. Here the computed results of the shallow water flow model are taken as the benchmark. As shown in Fig. 6, $E$ decreases from $t=4000 \mathrm{~s}$. At $t=9000 \mathrm{~s}, E$ is $10 \%$ lower, and the results predicted by the diffusion model presents the same matched flood extent state as the simulation of the shallow water flow model. It also proves that the accuracy of the diffusion model converges to the shallow water flow model.

The efficiency of the diffusion model is presented by comparing the computational times for the simulations as shown in Fig. 6. The results show that the diffusion model is more efficient because it helps reducing the computational run times (from $5084 \mathrm{~s}$ by the shallow water model to $157 \mathrm{~s}$ by the diffusion model) while the accuracy of the diffusion model converges to the shallow water model.

\section{Accuracy and efficiency of the adaptive scheme}

In this section, the accuracy and efficiency of the dynamically adaptive scheme are tested by comparing with the simulations on the uniform grids. The simulation is performed on the domain of digital terrain data $36000 \mathrm{~m} \times 36000 \mathrm{~m}$, generated from Shuttle Radar Topography Mission data source. The 

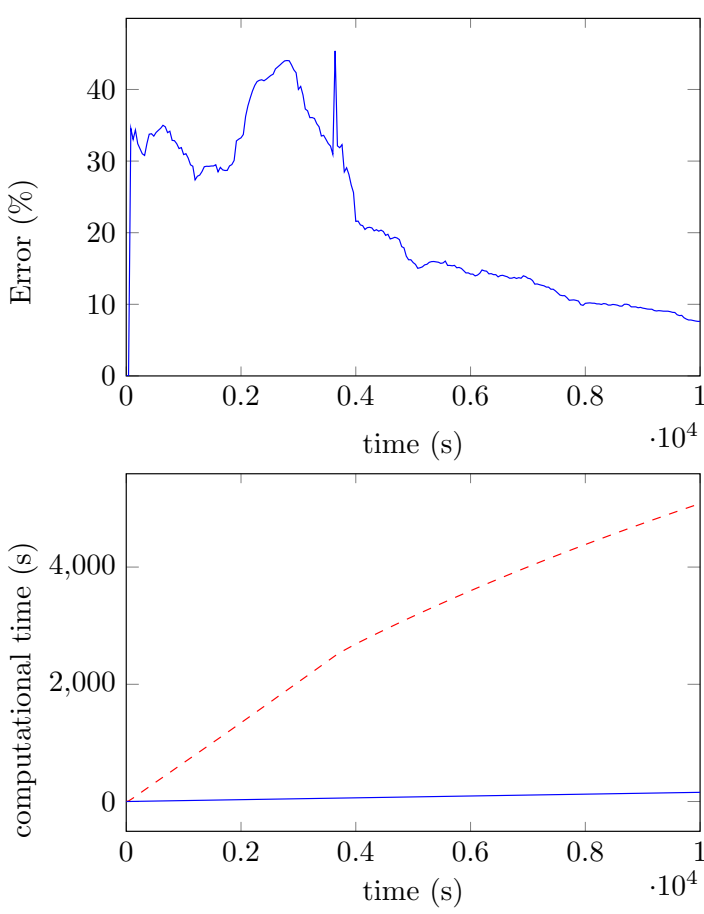

Fig. 6 The percentage errors of the diffusion model compared with the shallow water equations (top), and the comparison of the computational times (bottom) for the simulation of the diffusion model (solid) and the shallow water equations (dashed).

maximum tree grid level is 4 with resolutions $360 \mathrm{~m}$ $\times 360 \mathrm{~m}, 180 \mathrm{~m} \times 180 \mathrm{~m}, 90 \mathrm{~m} \times 90 \mathrm{~m}$, and $45 \mathrm{~m}$ $\times 45 \mathrm{~m}$, for levels $1,2,3$, and 4 , respectively; therefore, the initial grid level 1 has $100 \times 100$ cells. The topography values are provided for levels 1, 2 and 3, except the level 4 which are obtained automatically via the triangular planes approximation. The tree grid cells for children are designed as $2 \times 2$ for levels 1,2 , and 3 , while level 4 cells have no children.

The numerical experiment is simulated for $53 \mathrm{~h}$ with the water diffusion coefficient $50.625 \mathrm{~m}^{2} / \mathrm{s}$, and the parameters of the grid adaptivity are $\epsilon=0.1$ and $\xi=0.001$. The rainfall rate is set at $0.001 \mathrm{~m} / \mathrm{s}$ within the two rectangular regions which are the first region: $13500 \mathrm{~m} \leqslant x \leqslant 18000 \mathrm{~m}$ and $21150 \mathrm{~m}$ $\leqslant y \leqslant 25650 \mathrm{~m}$, and the second region: $18900 \mathrm{~m}$ $\leqslant x \leqslant 23400 \mathrm{~m}$ and $19800 \mathrm{~m} \leqslant y \leqslant 24300 \mathrm{~m}$ for the duration of $7200 \mathrm{~s}$. The domain boundaries are defined as the open boundary condition.

The 3D representation of the rainfall overland flow simulation for this experiment are presented at different times in Fig. 7, and the corresponding automatically adapted grids are shown in Fig. 8.
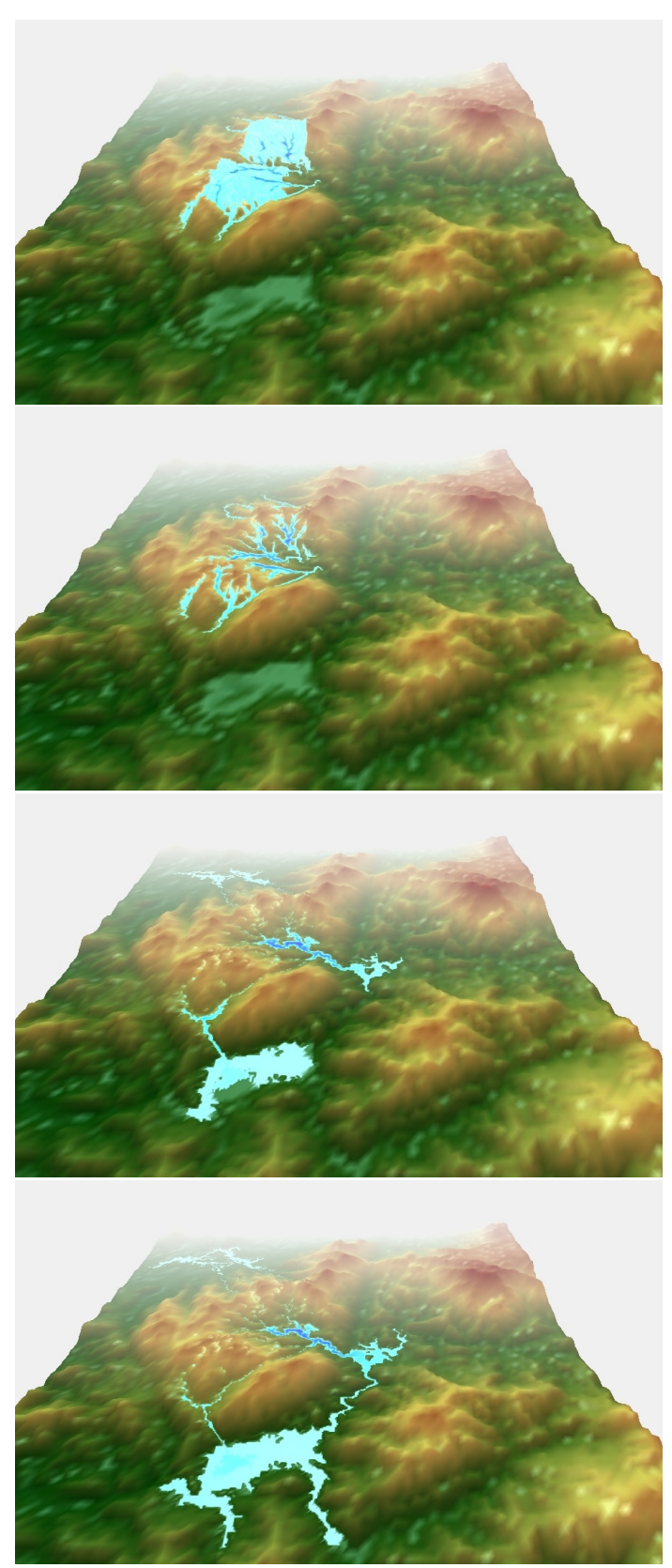

Fig. 7 The 3D representation of the adapted grids of the rain water overland flow simulation at $t=100,140$, 1400 , and $3180 \mathrm{~min}$ (from top to bottom).

The results show that the high-resolution areas are automatically obtained by the adaptivity technique in corresponding to the regions where the free surface gradients are steep and have water depths more than $0.001 \mathrm{~m}$, based on adaptivity parameters. The results also indicate that the diffusion model with the developed numerical scheme has a potential for 

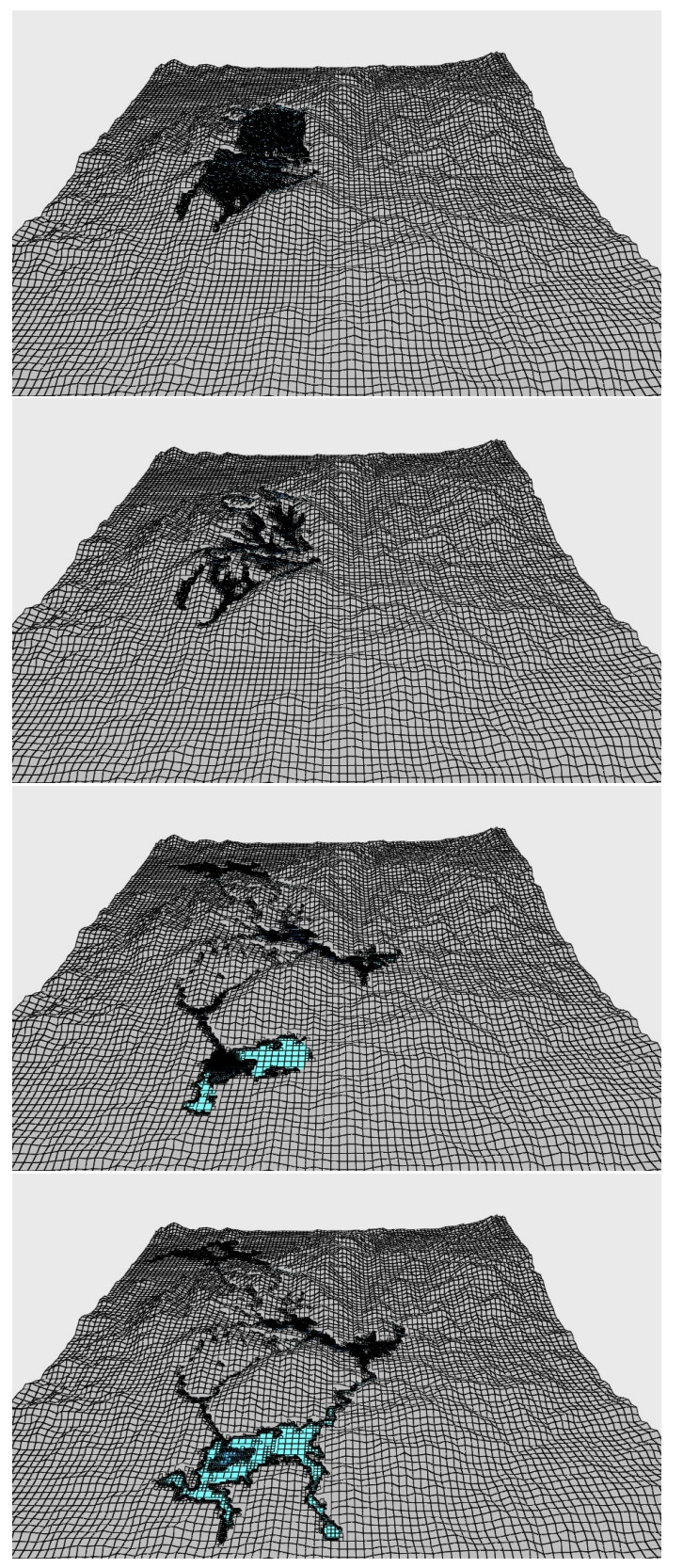

Fig. 8 The 3D representation of the adapted grids of the rain water overland flow simulation at $t=100,140$, 1400, and $3180 \mathrm{~min}$ (from top to bottom).

the simulation of the water flow in rugged terrain.

To check the accuracy and efficiency of the dynamically adaptive scheme we performed the following test. We compared the dynamically adaptive simulation with non-adaptive simulations on two uniform grids, level 1 grid (largest cells with $100 \times 100$ ) and level 4 grid (smallest cells with
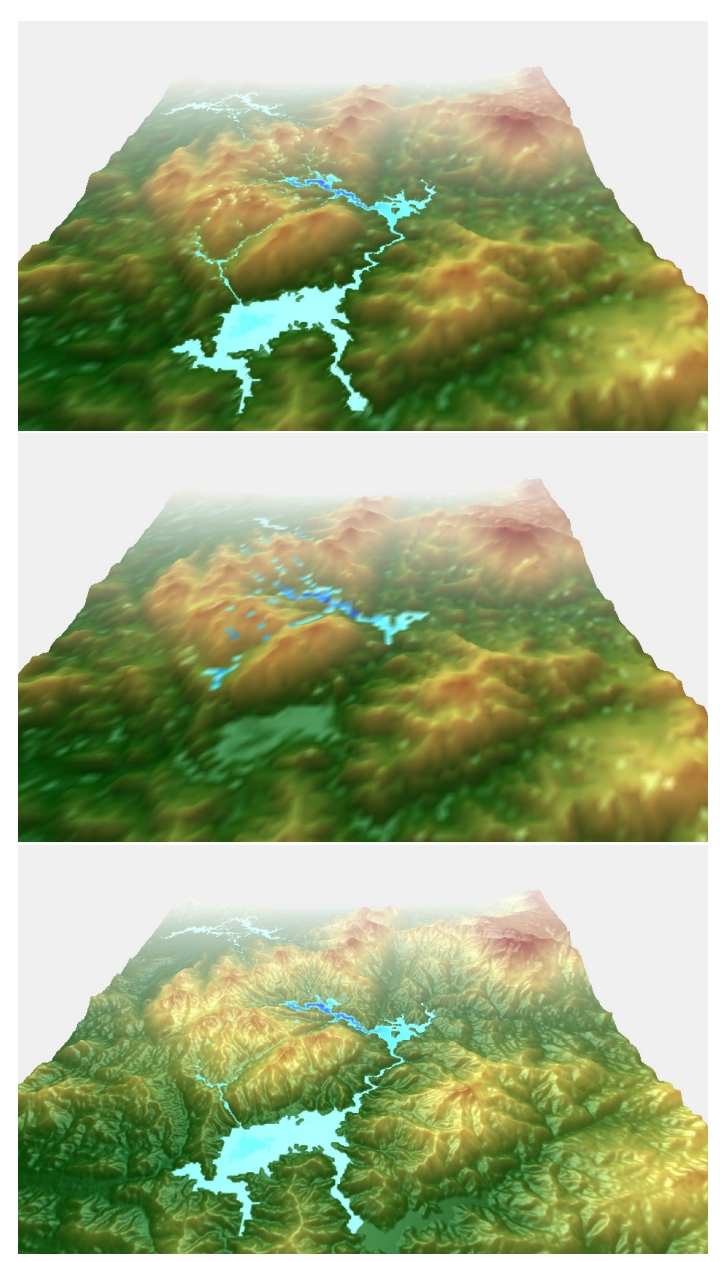

Fig. 9 The 3D representations of comparisons for adaptive grid (top), uniform coarsened grid $100 \times 100$ cells grid (centre), and uniform finest grid $800 \times 800$ cells grid (bottom) of the rainfall overland flow simulation at $t=$ $53 \mathrm{~h}$.

$800 \times 800$ cells). The 3D representations of the rainfall overland flow simulations at $53 \mathrm{~h}$ for this test are shown in Fig. 9. The results show that the simulation and the flow profiles obtained by the dynamically adaptive scheme are nearly the same as that obtained from the finest grid (level 4 grid), while the result from the largest cells (level 1 gird) is not acceptable. This shows the accuracy of the adaptive scheme. The efficiency of the adaptive scheme is presented by comparing the number of computational cells and the computational times for the simulations (Fig. 10). The results show that the adaptive scheme is more efficient because it helps reducing the computational cells (from 640000 cells by the finest grid to less than 35000 cells by the adaptive grid), and therefore reduces the 

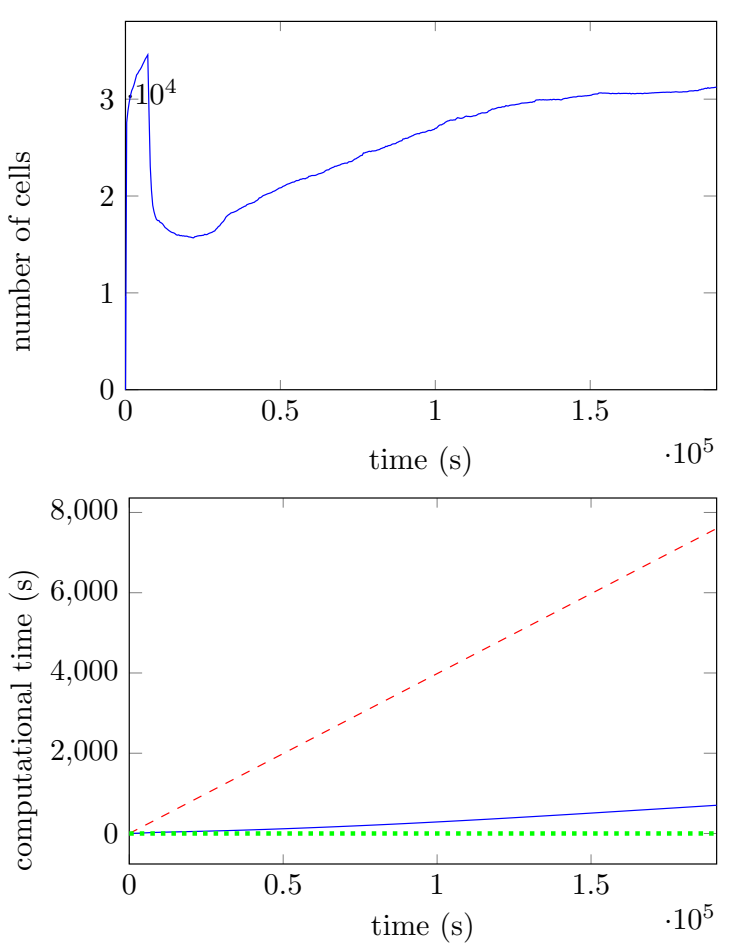

Fig. 10 The numbers of computational cells for adaptive grid (top), and the comparison of computational times (bottom) for adaptive grid (solid), the uniform small grid cells (dashed), and the uniform large grid cells (dotted).

computational run times (from $7600 \mathrm{~s}$ by the finest grid to $703 \mathrm{~s}$ by the adaptive grid) while keeping the same accuracy as that of the finest grid.

\section{CONCLUSIONS}

The simulations of rainfall overland flow in general have been developed based on shallow water equations. In this work, the algorithm has been constructed based on the diffusion model to simulate the inundation of rainfall in rugged terrain using the adaptive finite volume scheme. The algorithm applied the dynamically adaptive rectangular tree grid technique and together with the dynamic DDM. The comparisons of the developed simulation results with the uniform small and large cell size grids results reveal the advantages that it can reduce the number of the computational cells and the simulation run times without losing much accuracy in the results. This is an enormous efficiency advantage in terms of computational costs. The model could be improved by including other processes of rain-water over land as presented in Refs. 2,13, which can be very helpful for the flood risk assessments and water management.
Acknowledgements: We would like to thank the editors and referees for their valuable comments, which have significantly improved this paper. This study is supported by the Science Achievement Scholarship Thailand.

\section{REFERENCES}

1. Delestre O, Cordier S, James F, Darboux F (2008) Simulation of rain-water overland flow. In: Proceedings of the 12th International Conference on Hyperbolic Problems, Univ of Maryland, pp 537-46.

2. Mei X, Decaudin P, Hu BG (2007) Fast hydraulic erosion simulation and visualization on GPU. In: 15th Pacific Conference on Computer Graphics and Applications, Los Alamitos, pp 47-56.

3. Wang Y (2011) Numerical improvements for largescale flood simulation. PhD thesis, Newcastle Univ.

4. Santillana M (2008) Analysis and numerical simulation of the diffusive wave approximation of the shallow water equations. PhD thesis, Univ of Texas at Austin.

5. Dottori F, Todini E (2011) Developments of a flood inundation model based on the cellular automata approach: Testing different method to improve model performance. Phys Chem Earth 36, 266-80.

6. George DL (2011) Adaptive finite volume methods with well-balanced Riemann solvers for modeling floods in rugged terrain: application to the Malpasset dam-break flood (France, 1959). Int J Numer Meth Fluid 66, 1000-18.

7. Liang Q, Du G, Hall JW, Borthwick AG (2008) Flood inundation modeling with an adaptive quadtree grid shallow water equation solver. J Hydraul Eng 134, 1603-10.

8. Popinet S (2011) Quadtree-adaptive tsunami modelling. Ocean Dynam 61, 1261-85.

9. Audusse E, Bouchut F, Bristeau MO, Klein R, Perthame B (2004) A fast and stable well-balanced scheme with hydrostatic reconstruction for shallow water flows. SIAM J Sci Comput 25, 2050-65.

10. Yamaguchi S, Ikeda T, Iwamura K, Naono K, Ninomiya A, Tanaka K, Takahashi H (2007) Development of GIS-based flood-simulation software and application to flood-risk assessment. In: 2nd IMA International Conference on Flood Risk Assessment, Plymouth, UK.

11. Busaman A, Mekchay K, Siripant S, Chuai-Aree S (2015) Dynamically adaptive tree grids modeling for simulation and visualization of rain-water overland flow. Int J Numer Meth Fluid 79, 559-79.

12. Horritt MS, Bates PD (2001) Effects of spatial resolution on a raster based model of flood flow. $J$ Hydrol 253, 239-49.

13. Chevalier VL, Jaeger M, Mei X, Cournede PH (2007) Simulation and visualisation of functional landscapes: effects of the water resource competition between plants. $J$ Comput Sci Tech 22, 835-45. 